\title{
ACELEROGRAMAS Y ESPECTROS DE RESPUESTA EN MEDIOS: SUELO Y ROCA, BOYACÁ, COLOMBIA
}

\section{Comparison of accelerograms and response spectra in media: soil and rock, Boyacá, Colombia}

\author{
Luis Humberto Pinto Morales ${ }^{1}$, María del Carmen Fuentes Fuentes ${ }^{2}$ \\ 1. GEOSOFTMINE LTDA, Colombia. ${ }^{2}$ Universidad Pedagógica y Tecnológica de Colombia, \\ Escuela de Ingeniería Geológica, Grupo de Investigación INGEOFISICA, Colombia. \\ Email: ${ }^{1 h}$.pintomorales@gmail.com²maria.fuentes@uptc.edu.co
}

(Recibido febrero 20 de 2021 y aceptado junio 27 de 2021)

\section{Resumen}

El control de vibraciones implica la medición de estas perturbaciones en una voladura de producción. Si el nivel de vibraciones registrado fuera menor que el criterio de prevención, podrá incrementarse progresivamente la carga operante hasta que las intensidades de vibración fueran iguales al valor máximo admisible. En ese sentido, los análisis realizados en éste artículo sirven como estudios preliminares para establecer una tabla patrón de cargas máximas por tiempos de retardo en relación con la distancia a las estructuras a proteger, la velocidad y/o aceleración de la partícula.

Palabras clave: geofísica, geoeléctrica, sondeo eléctrico vertical.

\begin{abstract}
Vibration control involves the measurement of these disturbances in a production blasting. If the registered level of vibrations is less than the criterion of prevention, the operating load may be progressively increased until the vibration intensities were equal to the maximum allowable value. In this sense, the analyses in this article serves as preliminary studies to establish a standard table of maximum load for delay times in relation to the distance to the structures to protect, speed and/or acceleration of the particle.
\end{abstract}

Key words: geophysics, geoelectrical, vertical electrical sounding.

\section{INTRODUCCIÓN}

En este artículo se realiza una cualificación de los efectos de las voladuras en canteras, con el fin de reducir los niveles de las perturbaciones, en donde se establece un patrón de voladuras para los sitios de estudio; que cumpla con las normas ambientales vigentes a un costo aceptable. La utilización de explosivos, para fragmentar material rocoso, produce vibraciones en el terreno [7] y en una explotación minera, la perforación y voladura ocupa un lugar destacado, no solo por su peso dentro de los costos de operación, sino también por su influencia directa en los rendimientos de las fases del ciclo: carga, transporte y trituración.

Alteraciones producidas por las voladuras

Las voladuras efectuadas en las explotaciones mineras a cielo abierto; y subterráneas, generan vibraciones en el terreno, ya que una parte de la energía del explosivo se transforma en ondas elásticas que se trasmiten a través 
del medio rocoso circundante. Estas vibraciones pueden causar daños a estructuras próximas, edificaciones, obras subterráneas, etc.- e incluso molestias a personas que residan cerca de las áreas de trabajo-. Otra alteración que se genera, además de las proyecciones de fragmentos de roca, lo constituye la sobrepresión aérea (Ver Figura 1).

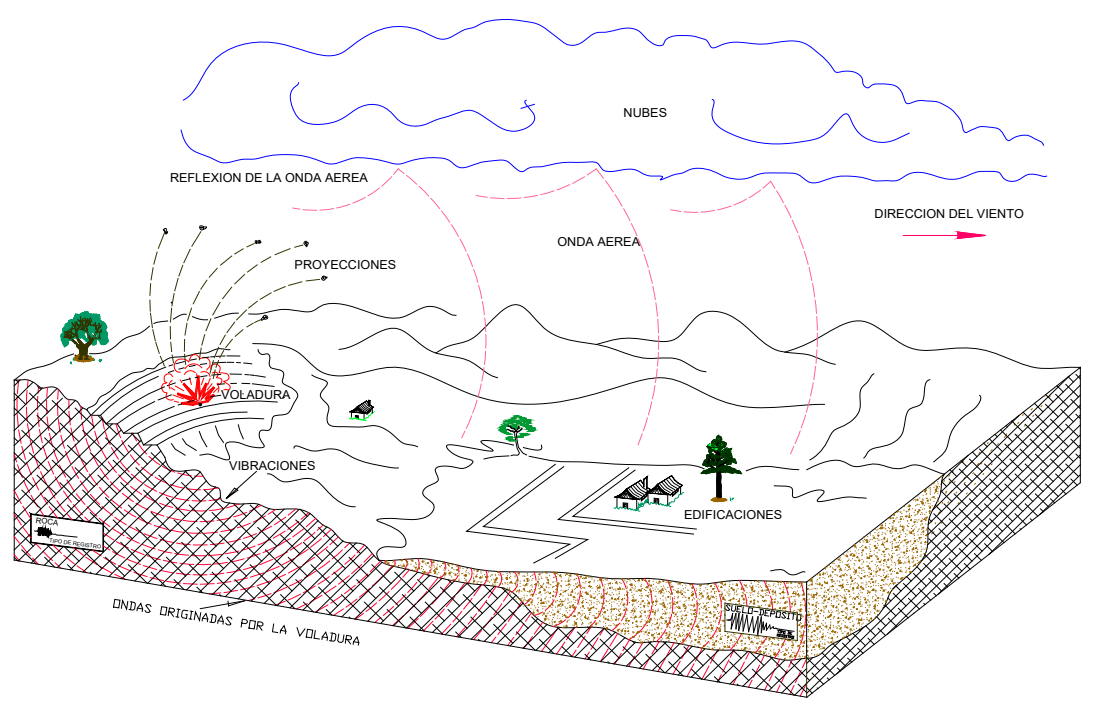

Figura 1. Perturbaciones originadas por las voladuras de rocas.

\section{Vibraciones terrestres}

En el campo de la ingeniería sísmica se distinguen dos grupos de ondas: las internas y las superficiales. Las ondas internas viajan en el macizo rocoso y se trasmiten de forma esférica en los mismos. Dentro de este grupo se distinguen las ondas primarias $\mathrm{P}$, que deforman la roca en la misma dirección de propagación estirándola y comprimiéndola como si fuera un muelle, y las ondas secundarias o S, que hacen vibrar la roca y viajan perpendicularmente a la dirección de propagación creando esfuerzos cortantes [6].

Las ondas superficiales se trasmiten generalmente por el terreno, si bien pueden propagarse por cualquier otra superficie donde entren en contacto dos materiales distintos: aire - agua, rocas de diferentes características etc. [8].

La sismología, como ciencia observacional, considera a las ondas superficiales Rayleigh y Love como un tipo particular de ondas sísmicas que se propagan paralelas a la superficie de un medio. Estas ondas tienen su amplitud máxima en la superficie libre y disminuye exponencialmente con la profundidad. Tienen una velocidad de trasmisión menor que las internas y sus frecuencias son más bajas, por lo que, en ocasiones pueden verse en las colas de los trenes de ondas que se generan en las voladuras, amortiguándose menos que las ondas internas y portando a una cierta distancia la mayor parte de la energía.

En los trenes de ondas reales, van superpuestas varias ondas independientes y no existe una sola frecuencia de vibración, sino un amplio margen de frecuencias con un valor más o menos dominante. No obstante, en ocasiones se asimila el paso de las ondas por un punto a un movimiento sinusoidal simple, cuyas ecuaciones de movimiento son:

$X=V_{\text {máx }} * 2 \pi * f$

$f=$ Frecuencia de vibración

$V_{\text {máx }}=D_{\text {máx }} * 2 \pi^{*} f$ 
Donde:

$V_{\text {máx }}=$ Velocidad de vibración máxima

$A_{\text {máx }}=$ Aceleración de vibración máxima

$A_{\text {máx }}=D_{\text {máx }} *\left(2 \pi^{*} f\right)^{2}$

$D_{\text {máx }}=$ Desplazamiento máximo del punto al paso de la onda.

Las frecuencias de las vibraciones dependen de las propiedades del macizo y del espesor y tipo de recubrimiento. En general, con rocas aflorantes y competentes, se tienen frecuencias altas $(20-80 \mathrm{~Hz})$, amplitudes bajas y amortiguación rápida.

En materiales de recubrimiento se tienen frecuencias bajas $(10-20 \mathrm{~Hz})$ y amplitudes elevadas. La Figura 2, es un ejemplo de frecuencias comunes en rocas [4].

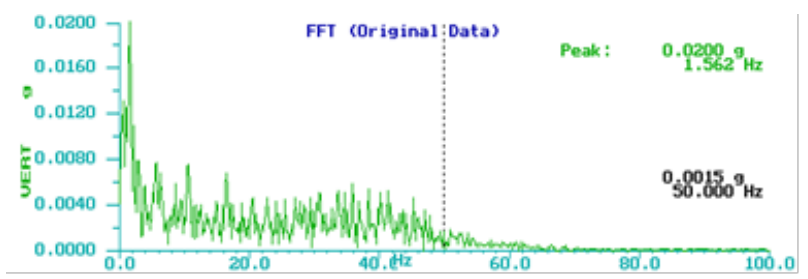

Figura 2. Espectro de frecuencia en función de la gravedad, (componente vertical).

\section{Estudio de vibraciones}

La fragmentación de las rocas por un explosivo incluye: la generación de una onda de tensión por la presión del terreno, el desarrollo de un sistema de grietas por la penetración de gases a alta presión y la liberación y aceleración de la masa rocosa fragmentada a una determinada velocidad [1].

La apertura de las grietas por la expansión de los gases es el mecanismo que más contribuye a la vibración del terreno, generando ondas superficiales que no tienen gran dispersión y que se disipan menos que las ondas internas. De esta manera, el objeto básico de un estudio de voladuras se concreta en dos puntos:
- Conocer la ley de propagación que relaciona cargas detonantes y distancias con la intensidad máxima de vibración generada.

- Conocer las frecuencias o periodos dominantes y el efecto de la superposición entre disparos.

\section{MATERIALES Y MÉTODOS}

Con los datos de frecuencias o periodos dominantes y empleando criterios definidos por la normativa vigente, se puede adoptar un límite de vibración o umbral adecuado que una vez introducido en la ley de propagación, permita calcular la carga máxima a detonar en cada distancia a la estructura a proteger.

Los parámetros, generalmente, utilizados para caracterizar la magnitud de la vibración son la velocidad y la aceleración de la partícula, por ser los que mejor se correlacionan con el riesgo de daños a estructuras.

La importancia de esta investigación radica, esencialmente, en el contraste entre la instalación de acelerógrafos en dos puntos determinados. Es decir, se trabajará en función de la aceleración máxima de la partícula utilizando datos suministrados por dos acelerógrafos ubicados en las canteras de calizas en las minas "Nobsa y Suescún". En donde, el acelerógrafo de Nobsa está ubicado sobre suelo y el de Suescún sobre roca.

\section{Instrumentación}

El acelerógrafo, es un instrumento diseñado para que se active en la zona de altas frecuencias del espectro de respuesta, en la cual domina la aceleración; mientras que, el sismógrafo es diferente en cuanto a su diseño, pues este responde, principalmente, en la zona de frecuencia intermedia, en la cual domina la velocidad.

En la Figura 3, se aprecia un acelerógrafo esquematizado como una caja resistente que está sujeta contra el medio por el cual se desplazan las ondas sísmicas; la caja se mueve exactamente igual que el suelo. Dentro de la caja se mueve un oscilador simple amortiguado [2]. 


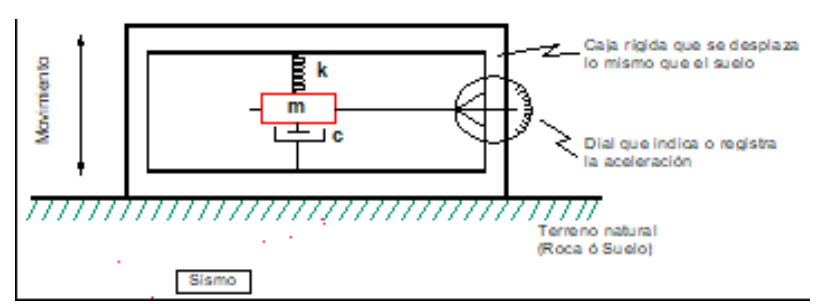

Figura 3. Oscilador simple.

Un oscilador simple es un sistema lineal de un grado de libertad, en el cual, la posición de la masa o cuyas características energéticas en función del tiempo, pueden presentarse en términos de una variable. En el caso de los sistemas mecánicos, esta variable fundamental es el desplazamiento, puesto que de éste se derivan la velocidad y la aceleración [3].

Este tipo de mecanismo proporciona un registro visual de la vibración producida por la voladura, de tal manera que, permite su análisis. Se basa en los cambios de voltaje producidos en el sensor, que son calibrados, amplificados y referenciados introduciendo una escala de tiempos [5]. El registro puede hacerse sobre un papel fotográfico o bien en un soporte magnético que posibilita un estudio más detallado con su posterior reproducción.

El acelerograma, es el registro gráfico que se logra al pasar las ondas sísmicas por el sitio donde se ha colocado un instrumento que las detecta y registra, este puede reflejar las tres componentes del movimiento ondulatorio (Ver Figura 4).

Las componentes del sistema ondulatorio son:

- Longitudinal L: mide la componente en la dirección voladura- registro.

- Transversal T: mide la componente perpendicular a la dirección de trasmisión de la onda de trasmisión.

- Vertical V: mide la componente perpendicular al plano de la superficie.

La aceleración de la partícula total se obtiene por la combinación de las tres componentes en el vector resultante usando la fórmula:

$a_{R}=V a_{L}^{2}+a_{T}^{2}+a_{V}^{2}$

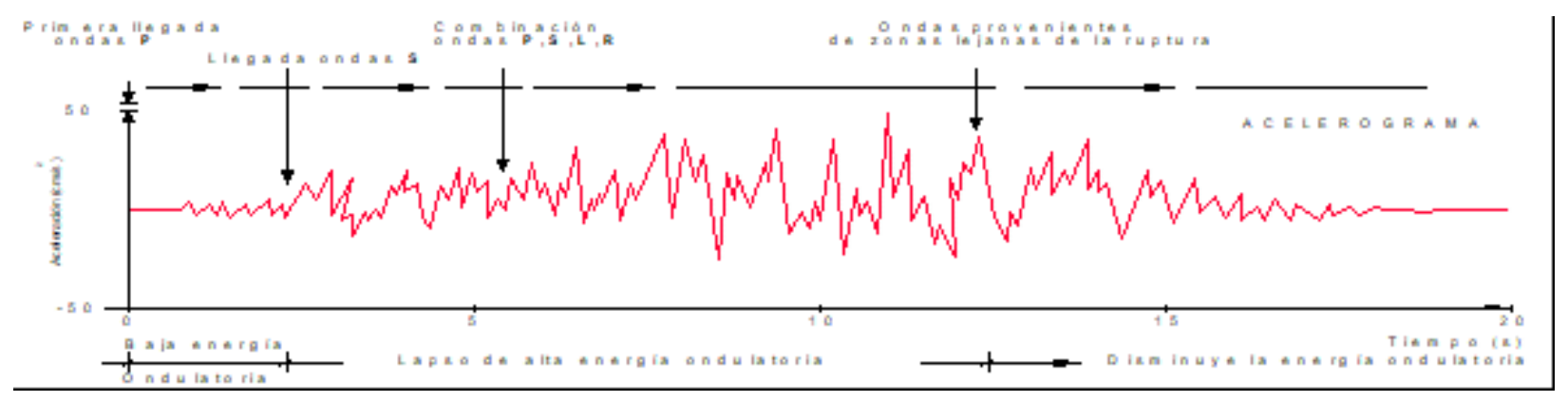

Figura 4. Acelerograma y fases de energía.

\section{Características instrumentales}

Acelerógrafo ubicado en la mina Nobsa (figura 5)

Código: K2- 395

Marca: KINEMETRICS

Modelo: ETNA

Tipo: DIGITAL
Sensibilidad: $0.1 \%$ de la escala total (2 Gales de aceleración del terreno)

Fecha de instalación: 24 febrero de 1996 Fecha de la última configuración: 20 de agosto de 2017

Altura: 2495 m.s.n.m. 


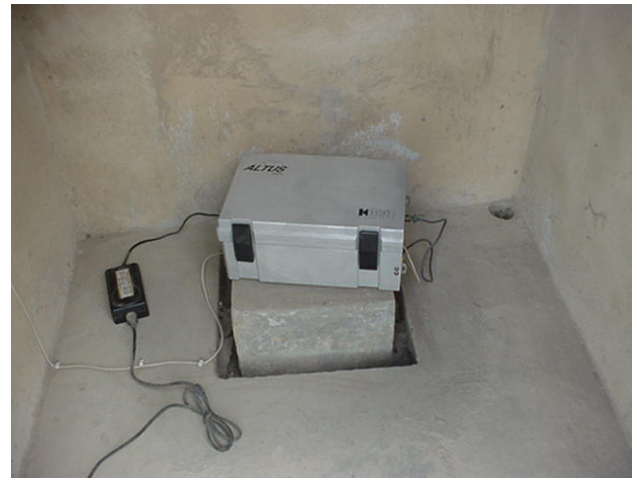

Figura 5. Acelerógrafo digital K-2_KINEMETRICS.

Acelerógrafo en la mina Suescún (Figura 6)

Código: CSUES

Marca: KINEMETRICS

Modelo: K- 2

Tipo: DIGITAL

Sensibilidad: $0.02 \%$ de la escala total (0.4 Gales de aceleración del terreno)

Fecha de instalación: 24 febrero de 1996

Fecha de la última configuración: 20 de agosto de 2017

Altura: 2508 m.s.n.m.

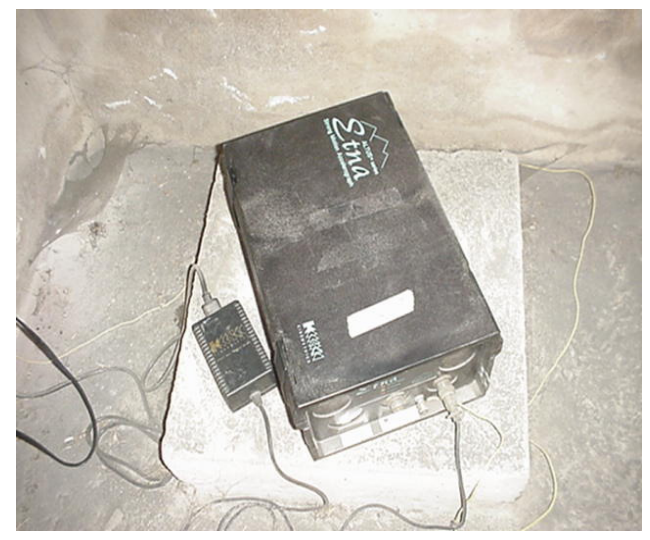

Figura 6. Acelerógrafo digital K-2_KINEMETRICS.

\section{Normativa colombiana relativa a vibraciones}

La norma vigente actual se plasma en el capítulo A.2, Sub. A.12.2 y A.12.3, de la Norma Sismo Resistente de 1998 (NSR-98), donde se muestra cómo y con qué parámetros se deben realizar los espectros de umbral de daño para diferentes zonas del país.
La finalidad de estos estudios es realizar una cualificación de los efectos de las voladuras y si éstas superan o no los límites establecidos (umbral de daño), con el objetivo de reducir y controlar niveles de perturbación.

Por otro lado, la normativa española resulta más conservadora, con un rango de seguridad igual a $0.1 \mathrm{~g}$, (Ver Figura 7), considerando a los eventos que sobrepasen de este valor, como precaución desde 0 - $80 \mathrm{~Hz}$ y velocidades de partículas que van desde $2.3-50 \mathrm{~mm} / \mathrm{seg}$.

El umbral de $1 \mathrm{~g}$, es considerado como daño para velocidades que van entre los 15 y $20 \mathrm{~mm} / \mathrm{seg}$ hasta superiores a $500 \mathrm{~mm} / \mathrm{seg}$ y frecuencias superiores a 100 $\mathrm{Hz}$.

\section{Espectro de umbral de daño}

Definido por la Norma Sismo resistente, (NSR-98), es un espectro que trabaja la aceleración máxima de la partícula en Gales, (g, ordenadas), en función del período dominante del sitio en segundos ( $T$, abscisas).

Este espectro fue desarrollado con la finalidad de comprobar o verificar que las estructuras y elementos estructurales, se mantengan dentro del rango elástico de respuesta cuando se presenten movimientos sísmicos o en nuestro caso, vibraciones productos de la voladura.

En las Tablas 1- 3, se observan los parámetros de cálculo empleados para ambos sitios de monitoreo según [9].

Espectros de umbral de daño para Nobsa y Suescún

Estos espectros se encuentran representados en escala aritmética en las Figuras 8 y 9; definidos para un coeficiente de amortiguamiento del $2 \%$ del crítico. Los gráficos muestran el espectro de la aceleración (Sad), en el eje de las ordenadas y en las abscisas, el período calculado en sus fases: T, To, Td y TI.

Según los datos obtenidos, el tope máximo seguro para los espectros de aceleración de Nobsa y Suescún, con base en la normativa colombiana, es de $\mathrm{Sad}=0.12 \mathrm{~g}$. 
Digitalización y análisis de acelerogramas

En la digitalización y análisis de los acelerogramas, se deben tener en cuenta los espectros de respuesta que están relacionados con el umbral de daño.

Se define como respuesta al conjunto de máximos valores de desplazamiento, velocidad o aceleración, que sobre un oscilador simple puede originar una capa aleatoria o determinista [10].

Tabla 1. Parámetros de cálculo.
El método de los espectros de respuesta presentados se emplea con mayor confiabilidad en términos de discriminación relativa de la respuesta sísmica similar de las áreas estudiadas.

En la Figura 10, se observa la superposición de los dos espectros.

\begin{tabular}{|c|c|}
\hline $\begin{array}{l}\text { PARAMETROS DE } \\
\text { UMBRAL DE DAÑO }\end{array}$ & NOMENCLATURA \\
\hline Aa & Coeficiente que representa la aceleración pico efectiva, para diseño. \\
\hline Ad & Coeficiente que representa la aceleración pico efectiva para el umbral de daño. \\
\hline $\mathbf{I}$ & Coeficiente de importancia, según grupo de uso asignado para cada edificación. \\
\hline S & Coeficiente de sitio, según el tipo de perfil de suelo. \\
\hline$\overline{\mathbf{s}}$ & Coeficiente de sitio, para ser empleado en el espectro elástico de umbral de daño ( $\mathrm{S}=1.25 * \mathrm{~S})$. \\
\hline Sad & $\begin{array}{l}\text { Valor de espectro de aceleraciones del umbral de daño, para un periodo de vibración dado. Máxima } \\
\text { aceleración horizontal para un umbral de daño, expresada como una fracción de aceleración de la } \\
\text { gravedad, para un sistema de un grado de libertad con un periodo de vibración T. }\end{array}$ \\
\hline $\mathbf{T}$ & Periodo de vibración del sistema elástico, en segundos. \\
\hline Td & $\begin{array}{l}\text { Periodo de vibración, en segundos, correspondiente a la transición entre la zona de aceleración } \\
\text { constante del espectro de umbral de daño, para periodos cortos y la parte descendente del mismo. }\end{array}$ \\
\hline To & $\begin{array}{l}\text { Periodo de vibración, en segundos, correspondiente al inicio de la transición de la aceleración pico } \\
\text { efectiva constante hasta el periodo de vibración Td. }\end{array}$ \\
\hline TL & $\begin{array}{l}\text { Periodo de vibración, en segundos, correspondiente a la transición entre la zona de aceleración } \\
\text { constante del espectro de diseño, para periodos largos. }\end{array}$ \\
\hline g & Aceleración debida a la gravedad (g). \\
\hline
\end{tabular}

Tabla 2. Datos obtenidos según NSR-98.

\begin{tabular}{cccccccccc}
\hline SECTOR & $\begin{array}{c}\text { TIPO DE } \\
\text { PERFIL DE } \\
\text { SUELO }\end{array}$ & Aa & Ad & I & S & $\overline{\mathbf{S}}$ & To & Td & TL \\
\hline NOBSA & $\mathrm{S}_{2}$ & 0.30 & 0.04 & 1.0 & 1.2 & 1.5 & 0.25 & 0.75 & 2.88 \\
\hline SUESCUN & $\mathrm{S}_{1}$ & 0.30 & 0.04 & 1.0 & 1.0 & 1.25 & 0.25 & 0.625 & 2.4 \\
\hline
\end{tabular}


Tabla 3. Fórmulas para el cálculo del umbral de daño en los sectores de estudio.

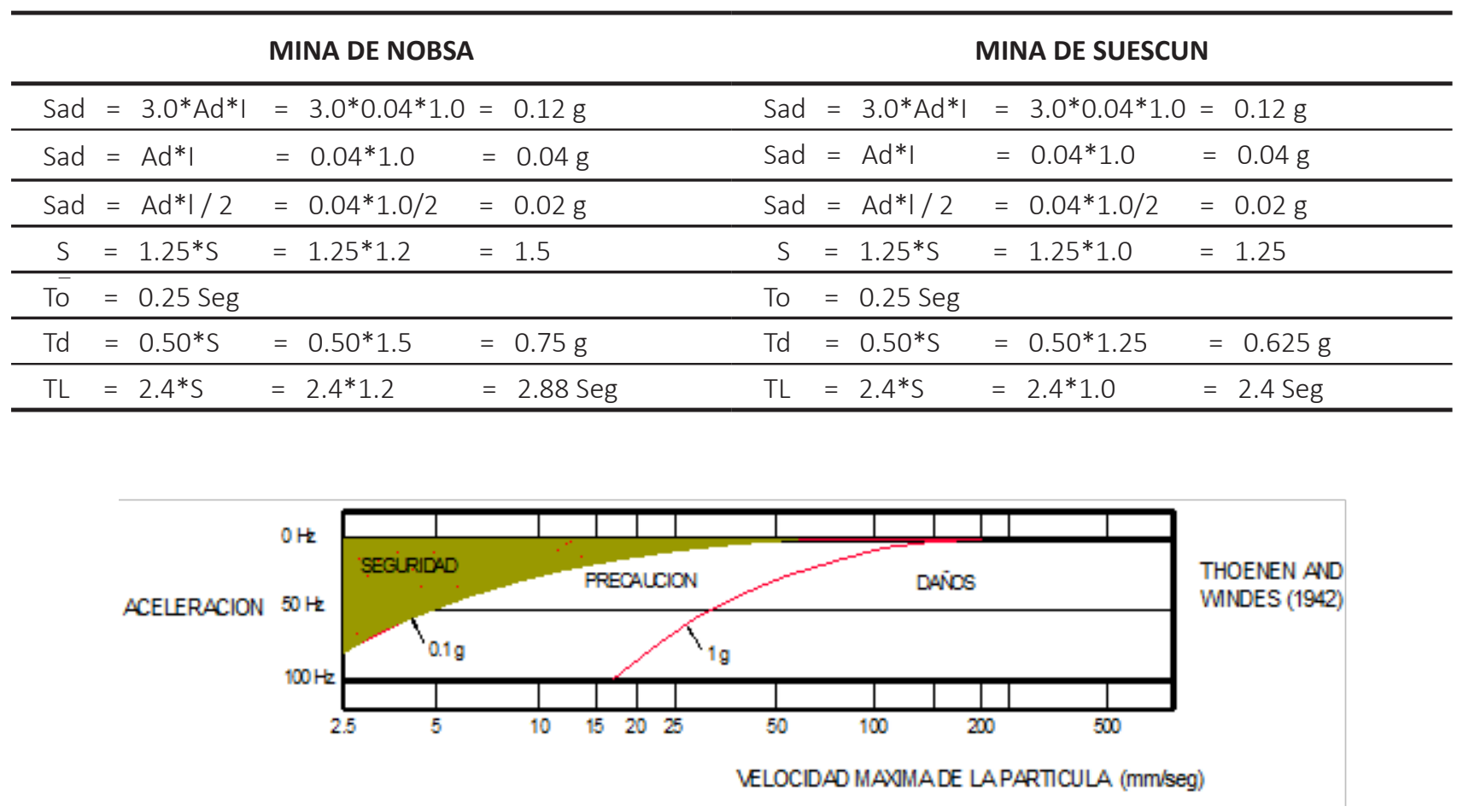

Figura 7. Legislación española vigente que se recoge en el reglamento general de normas básicas de seguridad minera, Instituto Tecnológico Geominero de España, ITGE.

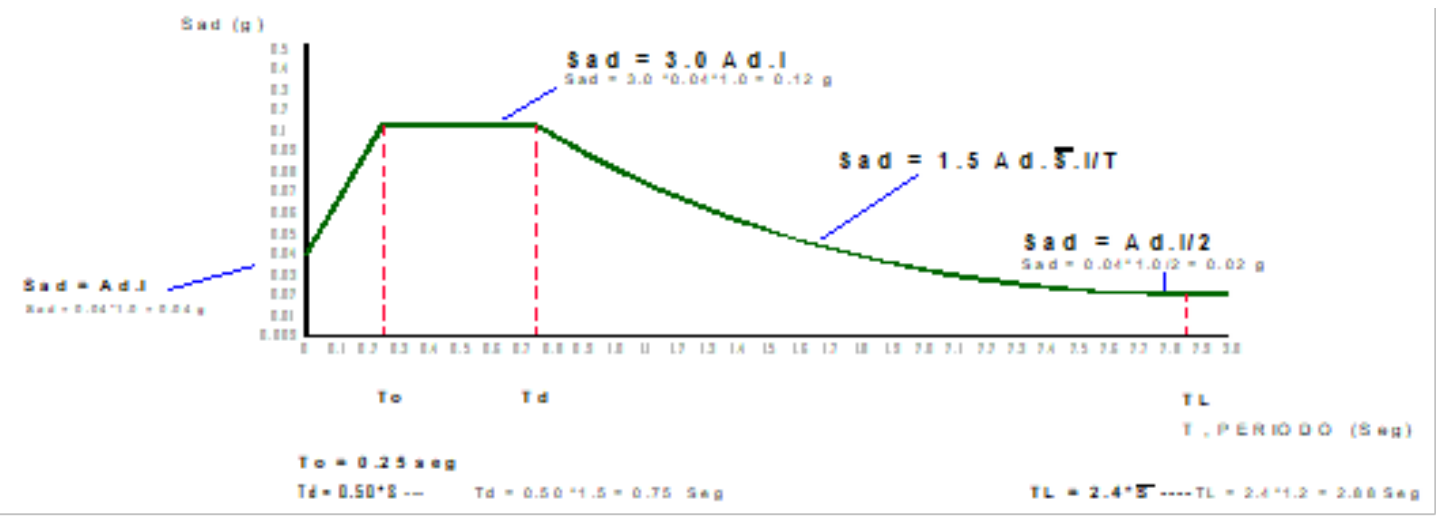

Figura 8. Aceleración espectral (g) vs periodo dominante (seg). 


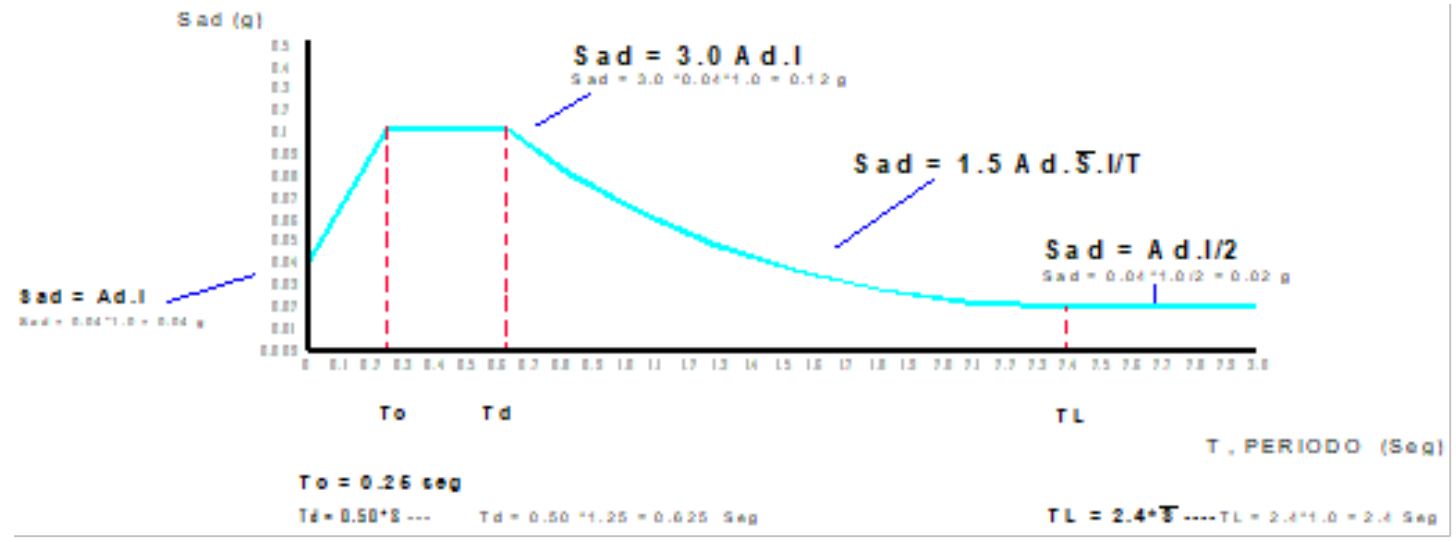

Figura 9. Aceleración espectral (g) vs periodo dominante (seg).

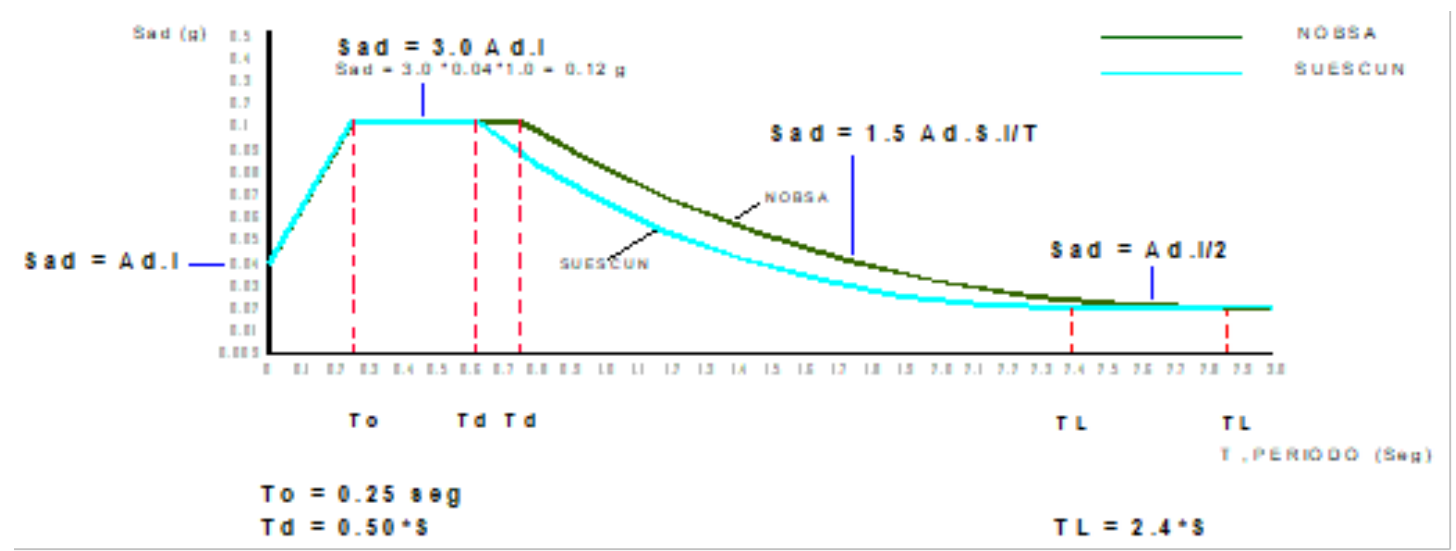

Figura 10. Aceleración espectral (g) vs periodo dominante (seg).

\section{RESULTADOS}

Este proyecto trabajó en función de la aceleración, sin embargo, en otros proyectos se adelantan estudios basados, fundamentalmente, en la velocidad de la partícula. En este caso, los umbrales para controlar las perturbaciones se establecen en función de la frecuencia de vibración dominante y del tipo de estructura a proteger, utilizando como parámetro de medida la velocidad con que llega la partícula a la edificación.

En los estudios preliminares se determina el comportamiento sísmico del terreno mediante la definición de la ley de propagación, realizando voladuras con diferentes cargas instantáneas y registros en posiciones diferentes que cubran el área de interés. Al conocer la frecuencia dominante, el tipo de estructura y consecutivamente, el criterio de daños, con la ley de propagación, se elabora la tabla de cargas máximas operantes por tiempos mínimos de retardos en función de la distancia. En seguida, se analizan los acelerogramas y se calculan los espectros de umbral de daño en escala Log - Log.

Los programas de análisis están desarrollados en esta escala con el fin de eliminar ondas no deseadas, caracterizando así las vibraciones dentro de un rango del espectro de umbral de daño expuestos anteriormente para Nobsa y Suescún, en escala aritmética. 


\section{Sector Nobsa}

El acelerógrafo está ubicado en suelo con una sensibilidad de $0.1 \%$ de la escala total, con amortiguamiento del 2 y el $5 \%$ del crítico. Los espectros de las tres componentes presentan una trayectoria similar, donde las mayores aceleraciones se presentan para periodos de $0.25 \mathrm{seg}$ y frecuencias de $4 \mathrm{~Hz}$ aproximadamente, (Ver Figuras 11 16 y Tabla 4).

\section{Sector Suescun}

El acelerógrafo está ubicado en roca con una sensibilidad de $0.02 \%$ de la escala total, con amortiguamiento del 2 y el $5 \%$ del crítico.

Los espectros de las tres componentes (L, longitudinal; $\mathrm{V}$, vertical y $\mathrm{T}$, transversal); presentan una trayectoria similar, donde las mayores aceleraciones se observan para periodos de 0.05 seg y frecuencias de $20 \mathrm{~Hz}$ aproximadamente.

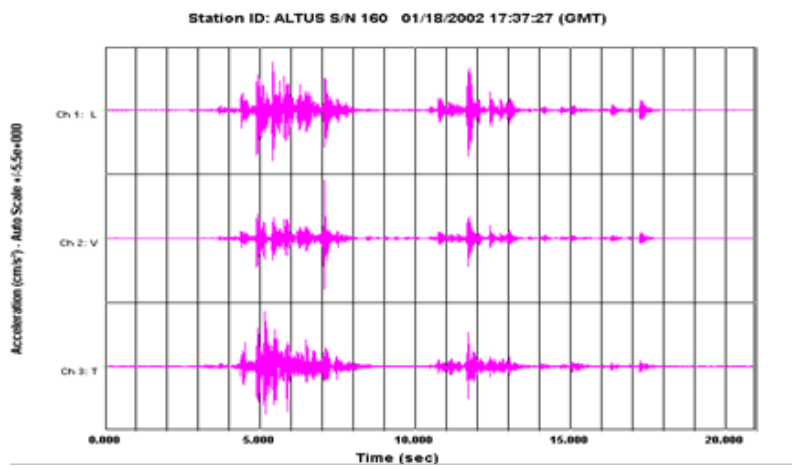

Figura 11. Acelerogramas de aceleración (g) vs tiempo (seg) en las tres componentes.

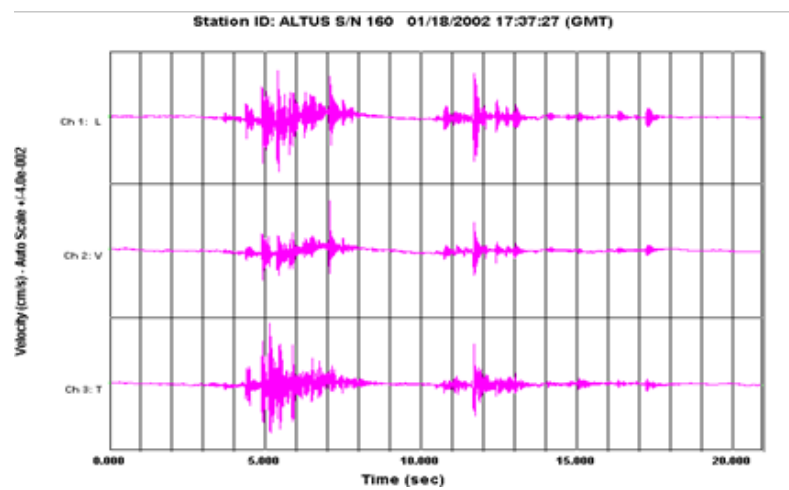

Figura 12. Velocidad vs tiempo en las tres componentes.

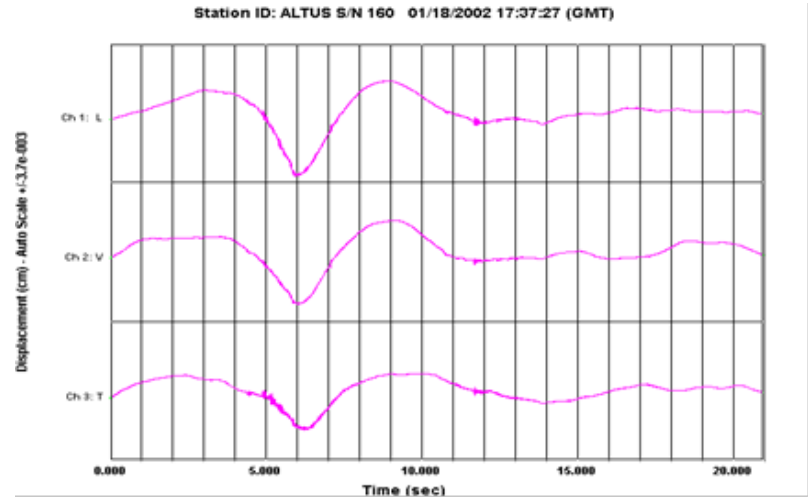

Figura 13. Desplazamiento vs tiempo en las tres componentes.

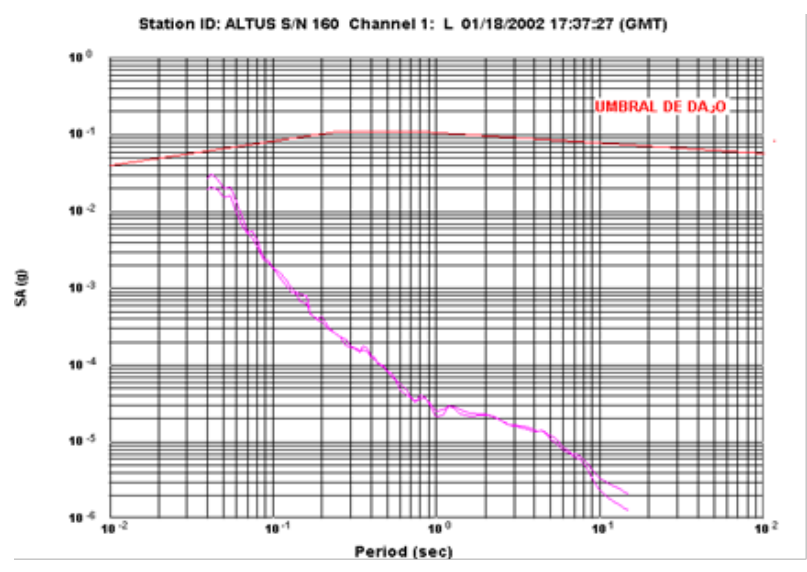

Figura 14. Espectro de aceleración vs periodo componente longitudinal.

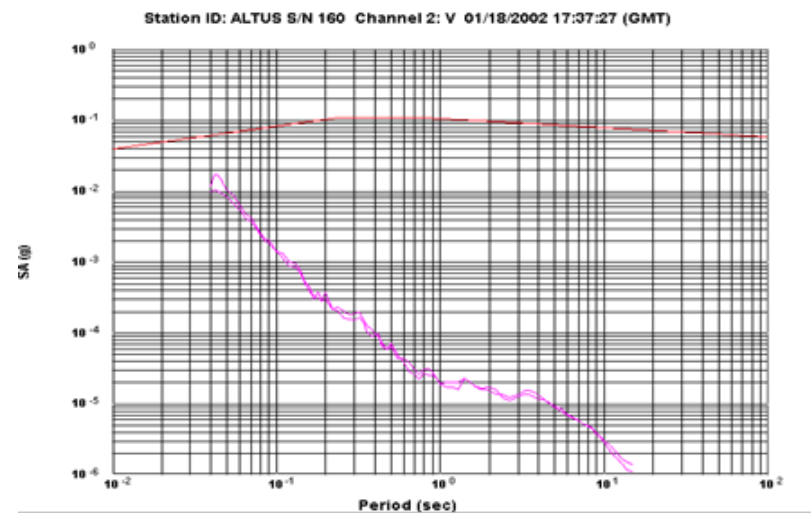

Figura 15. Espectro de aceleración vs periodo componente vertical. 


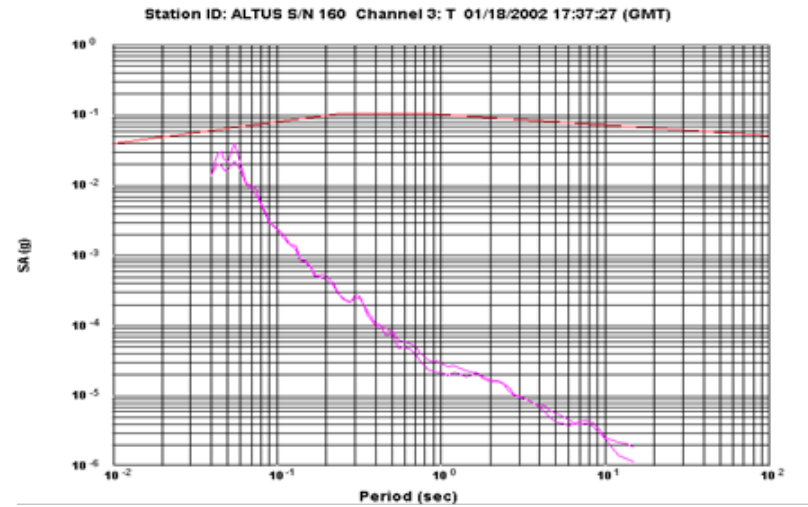

Figura 16. Espectro de aceleración vs periodo componente transversal.

Tabla 4. Aceleraciones máximas en las tres componentes: Longitudinal (E-W), Vertical (V) y Transversal (N-S).

\begin{tabular}{ccccccc}
\hline \multirow{2}{*}{ ESTACIÓN } & \multirow{2}{*}{ FECHA } & HORA & LONG. & VERTIC. & TRANS. & \multirow{2}{*}{ EVENTO } \\
& & & $\mathbf{g}$ & $\mathbf{g}$ & $\mathbf{g}$ & \\
\hline \multirow{2}{*}{ Nobsa } & $1 / 18 / 2002$ & $17: 37: 27$ & 4.978 & $-10.367 *$ & -7.534 & Voladura \\
\hline
\end{tabular}

\section{CONCLUSIONES}

Los espectros de respuesta son herramientas de amplio uso en la ingeniería sísmica tanto en la microzonificación de un área, como en la caracterización de vibraciones naturales (sismos) o artificiales (voladuras, tráfico pesado, etc.), porque han permitido pasar del dominio del tiempo al dominio de la frecuencia o del periodo, los cuales realizan una comparación entre los espectros de respuesta y de umbral de daño.

Para evaluar los efectos de las voladuras sobre viviendas o estructuras cercanas a los frentes de explotación de las minas Nobsa y Suescún, se comparan los espectros de respuesta, para aceleraciones máximas en los tres componentes de la onda, con el espectro de umbral de daño, para comprobar si no exceden los límites establecidos por el código de construcción sismo resistente (NSR-98).

Los espectros de aceleración espectral vs periodo dominante del sitio para tres canales longitudinal $L$, vertical V, y transversal T, expuestos en las Figuras 14 - 16 y $20-22$, permiten ilustrar que ninguno supera el umbral de daño del código colombiano de construcción sismo resistente, estando por debajo del valor expuesto por la norma española, $(0.1 \mathrm{~g})$.

La onda aérea para Nobsa y Suescún, está en el orden de 70 a 80 db (decibeles), muy por debajo de lo permitido dentro del margen de seguridad establecido por normas internacionales el cual es de $130 \mathrm{db}$, (Ver Figuras 17- 22 y Tabla 5).

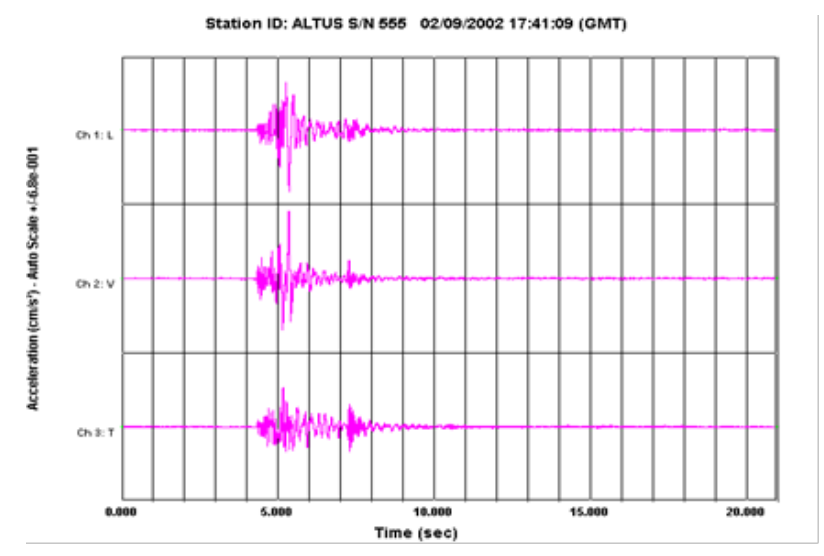

Figura 17. Acelerogramas de aceleración vs tiempo en las tres componentes.

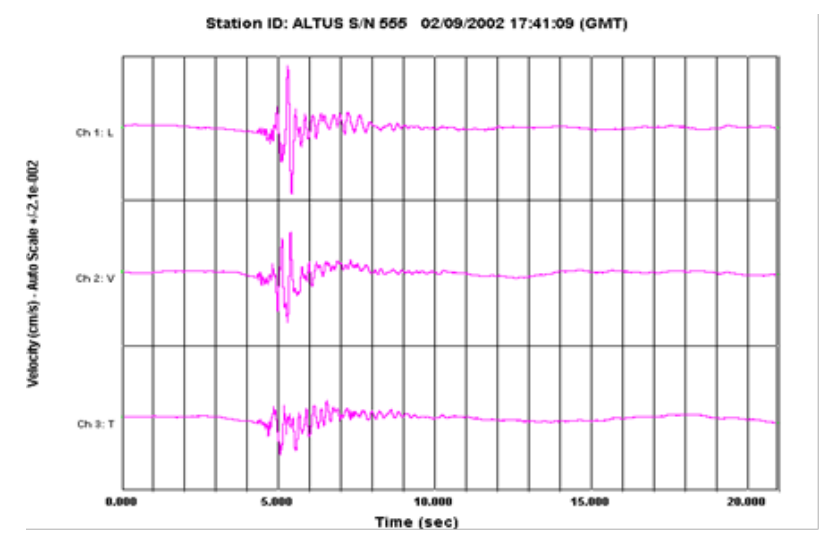

Figura 18. Velocidad vs tiempo en las tres componentes. 


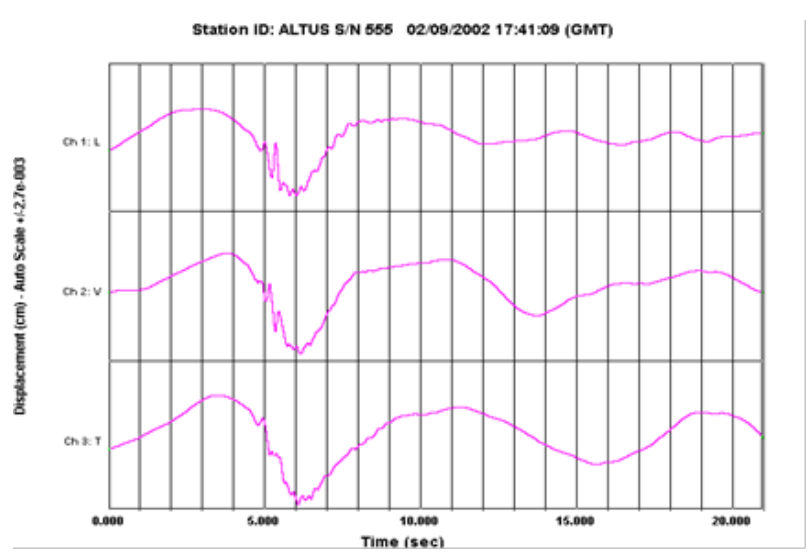

Figura 19. Desplazamiento vs tiempo en las tres componentes.

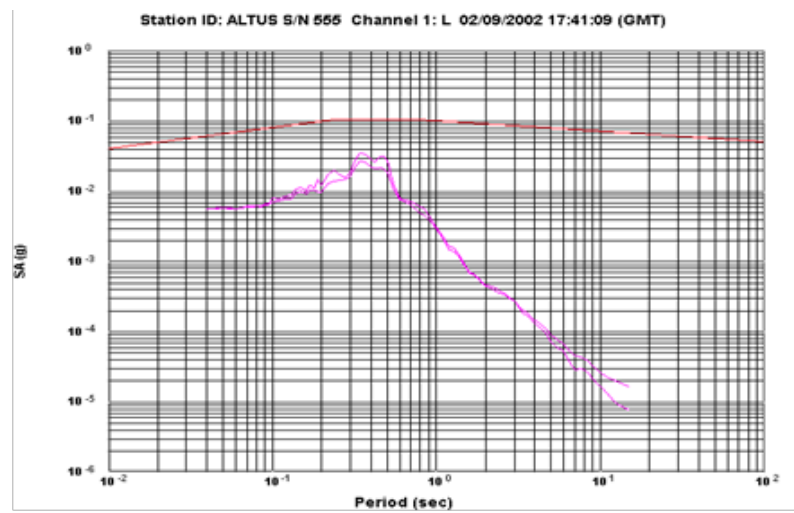

Figura 20. Espectro de aceleración vs periodo componente longitudinal.

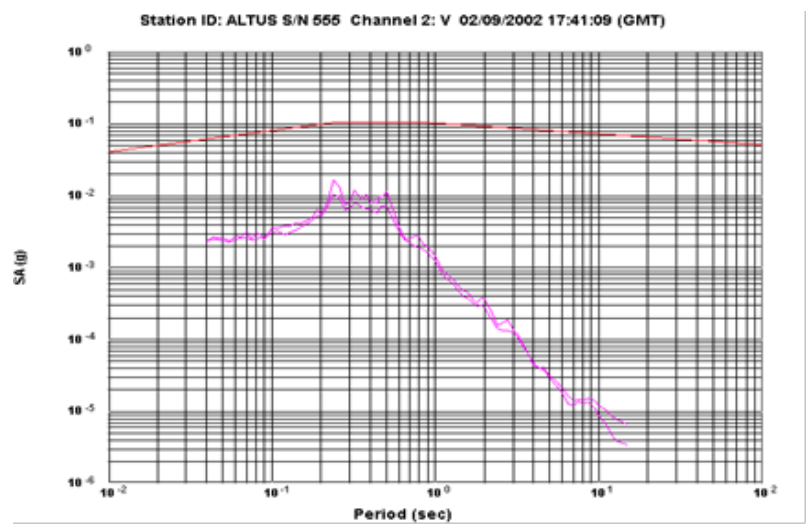

Figura 21. Espectro de aceleración vs periodo componente vertical.

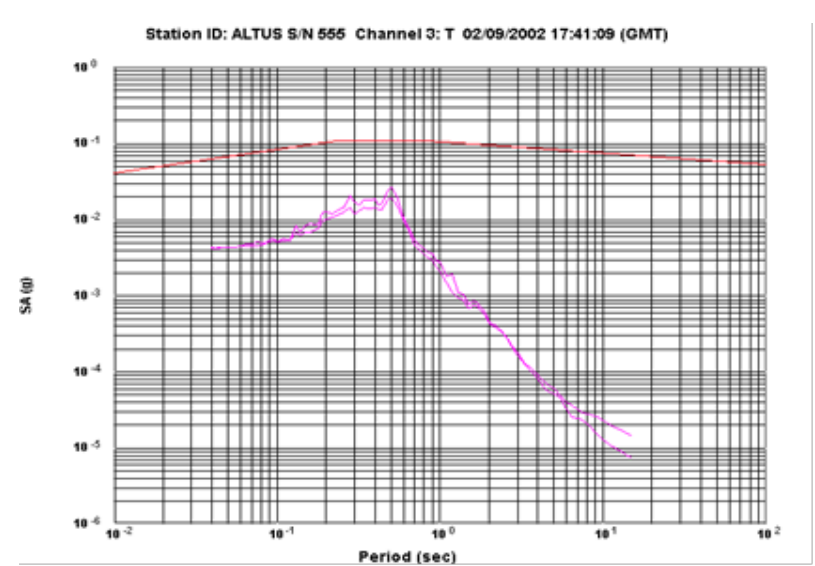

Figura 22. Espectro de aceleración vs periodo componente transversal.

Tabla 5. Aceleraciones máximas en las tres componentes: Longitudinal (E-W), Vertical (V) y Transversal (N-S).

\begin{tabular}{lcccccc}
\hline \multirow{2}{*}{ ESTACION } & FECHA & HORA & L ONG. & VERTIC. & TRANS. & \multirow{2}{*}{ EVENTO } \\
& & & $\mathbf{g}$ & $\mathbf{g}$ & $\mathbf{g}$ & \\
\hline Suescún & $02 / 09 / 2002$ & $17: 41: 09$ & 0.724 & 0.347 & $1.200 *$ & Voladura \\
\hline \multicolumn{6}{c}{ REFERENCIAS }
\end{tabular}

[1] P. Allard "Etude des vibrations Engendrées par les Tirs de Mines", Scetaroute, Février, 1986.

[2] R. C. Barkley, et al. Ground and Air Vibrations Caused by Surface Blasting, N.T.I.S., 1983.

[3] G. A. Bollinger, Blast Vibration Analysis, 1971.

[4] J. M. Esteves, "Control of Vibrations caused by Blasting", Memoria 498, Laboratorio Nacional de Engenharía Civíl, Lisboa, 1978.

[5] K. G. Hinzen, et al. A New Approach to predict and Reduced Blast Vibration by Modelling of Acelerograms and Using a New Electronic Initiation System. S. E. E., 1987.

[6] I.T.G.E. Manual de restauración de terrenos y evaluación de impactos ambientales en minería. España, 1994.

[7] J. Lleras Villaveces, Metodología para el análisis e interpretación de vibraciones ambientales. Tesis de Maestría. Ing. Civil. Universidad de los Andes, 1995.

[8] C. López Jimeno, Manual de voladura - Manual de minería en túnel y a cielo abierto, ETSIM Madrid, 
España. 1996.

[9] Normas Colombianas de Diseño y Construcción Sismo-Resistente (NSR-98).

[10] R. Tenreyro Pérez \& C. Rafca, Fundamentos de la prospección sísmica. La Habana. Cuba, 1988. 